\title{
THERMO-MECHANICAL EVOLUTION OF SHAPE MEMORY ALLOYS
}

\author{
BY \\ PIERLUIGI COLLI (University of Pavia, Italy) \\ MICHEL FRÉMOND (Laboratoire Central des Ponts et Chaussées, Paris, France) \\ AND \\ AUGUSTO VISINTIN (University of Trento, Italy)
}

\begin{abstract}
In certain alloys, after deformation the original shape can be recovered just by thermal means. This shape memory behaviour can be interpreted as the effect of an austenite-martensite phase transition. We model this phenomenon by assuming the free energy to depend on the volumetric proportions of the different phases.

The thermo-mechanical evolutions of a three dimensional body of such a material is here modelled in the framework of a second gradient theory. A variational formulation in Sobolev spaces is given; existence and uniqueness of the solution are proven by means of a fixed point argument.
\end{abstract}

1. Introduction. Some metallic alloys exhibit the following unusual behaviour: they can be permanently deformed (up to $8 \%$ under traction), but they totally recover their initial shape just by thermal means. This phenomenon is called shape memory, and can be interpreted as the effect of an austenite-martensite phase transition $[1,7$, $12,13]$.

At a microscopic scale, such an alloy is composed by a mixture of martensitic and austenitic crystals. We shall deal with this substance only at a macroscopic scale, and then assume that the phases coexist at each point. At a microscopic scale the austenite phase is homogeneous and presents higher symmetry, while the martensite phase exhibits less symmetry and is internally twinned, that is, it is formed of several variants related by symmetry (references can be found in $[3,14]$ ). For the sake of simplicity, we shall also suppose that just two martensitic variants are present, besides the austenite; however, this is sufficient to provide a consistent and exhaustive description of the peculiar behaviour of shape memory alloys.

Here we deal with the three dimensional model introduced in [8], taking the temperature, the macroscopic deformation and the volumetric proportions of austenite and martensite as state variables. As far as we know, all other macroscopic models using the same variables are in a single dimension of space and involve complicated

Received April 5, 1988.

Work supported by M. P. I. (fondi per la ricerca scientifica) and by I. A. N. of C. N. R., Pavia

(C) 1990 Brown University 
free energy expressions $[1,2,7,16]$. However, there are several other approaches accounting for microscopic properties [3,14]. Our model is based on simple and well-known expressions for the free energies of the phases, and the phase proportions are here regarded as thermodynamic quantities. The total free energy is obtained by summing the weighted free energies of the different phases and a contribution due to the mixture. This mixture free energy is very simple and expresses the compatibility condition that each of the proportions ranges between 0 and 1 , and that their sum is equal to 1 . Other examples of mixture free energies can be found in $[4,9,10]$.

Another peculiar aspect of our model is that it describes the mechanical action by means of a second gradient theory [11]; this is necessary if one wants to account for any mechanically induced phase transition.

The basic mechanical and thermodynamical equations are presented in Sec. 2. In Sec. 3 we give a variational formulation of this problem in Sobolev space. Then we prove the existence and uniqueness of the solution by means of an argument based on the Banach fixed point theorem.

2. The model. The shape memory effect depends essentially on the temperature and the phase composition, which can be modified by either thermal or mechanical actions. Here we shall denote by $\beta_{1}$ and $\beta_{2}$ the local proportions of the two martensitic variants, and by $\beta_{3}$ that of austenite.

The mechanical actions are exerted mainly on surfaces: they are surface tractions, surface couples, and surface double forces; all of them are to be accounted for. As is well known, the classical first gradient theory for the internal forces allows neither surface double forces nor surface couples. In order to take into account the latter we need a second gradient theory [11]. However, here we shall use just the most simple formulation of such a theory. Also for the sake of simplicity, we shall assume deformations to be small. In this framework the generalization to large deformations is straightforward (unpublished result of the second author).

2.1 Equilibrium equation. In the following we shall denote by $i, j, l, k$ some indexes with range $\{1,2,3\}$ and use the repeated index convention for summation. In the second gradient theory [11], the virtual power of the interior forces is defined by

$$
\begin{gathered}
\mathscr{P}_{I}:=-\int_{\mathscr{Z}}\left\{\sigma_{i j} \varepsilon_{i j}(\mathbf{v})+\mathbf{M g r a d} \operatorname{div} \mathbf{v}\right\} d x, \\
\varepsilon_{i j}(\mathbf{v}):=\frac{1}{2}\left(v_{i, j}+v_{j, i}\right),
\end{gathered}
$$

for any smooth domain $\mathscr{Z}$ and any velocity $\mathbf{v}$. The interior forces are the usual stresses $\sigma_{i j}$ and a vector $M$. Denoting by $\partial \mathscr{D}$ the boundary of $\mathscr{D}$ and integrating by 
parts, we obtain (see, for instance, [11], Appendix)

$$
\begin{aligned}
\mathscr{P}_{I}= & \int_{\mathscr{D}}\left(\sigma_{i j, j}-M_{l, l j} \delta_{i j}\right) v_{i} d x \\
& -\int_{\partial \mathscr{D}}\left\{\sigma_{i j} n_{j}-M_{l, l} n_{i}+n_{i}\left(M_{j} n_{j}\right)\left(\Delta_{p} n_{p}\right)-\Delta_{i}\left(M_{j} n_{j}\right)\right\} v_{i} d \Gamma \\
& -\int_{\partial \mathscr{D}}\left(M_{j} n_{j}\right) \varepsilon_{\mathbf{n n}}(\mathbf{v}) d \Gamma,
\end{aligned}
$$

where $\mathbf{n}=\left(n_{i}\right)$ is the outward normal unit vector to $\partial \mathscr{D}, \varepsilon_{\mathbf{n n}}=\varepsilon_{i j}(\mathbf{v}) n_{i} n_{j}$ is the normal elongation velocity and the $\Delta_{j}$ 's are the tangential derivatives defined by

$$
\Delta_{j} \varphi:=\varphi_{, j}-n_{j}\left(n_{p} \varphi_{, p}\right) .
$$

It is natural to define the virtual power of the external forces by

$$
\mathscr{P}_{E}=\int_{\mathscr{D}} \mathbf{f v} d x+\int_{\partial \mathscr{D}} \mathbf{T v} d \Gamma+\int_{\partial \mathscr{D}} N \varepsilon_{\mathrm{nn}}(\mathbf{v}) d \Gamma .
$$

Here $\mathbf{f}$ denotes a volume force. The local contact forces are the usual surface traction $\mathbf{T}$ and a normal surface double force $\mathbf{N}$, which causes a normal elongation.

The quasi-static equilibrium equations are provided by the principle of virtual power

$$
\forall \mathscr{D}, \quad \forall \mathbf{v}, \quad \mathscr{P}_{I}+\mathscr{P}_{E}=0 .
$$

They are

$$
\sigma_{i j, j}-M_{l, l j} \delta_{i j}+f_{i}=0
$$

or equivalently

$$
\tau_{i j, j}+f_{i}=0, \quad \text { in } \mathscr{D},
$$

with $\tau_{i j}:=\sigma_{i j}-M_{l, l} \delta_{i j}$, and

$$
\begin{gathered}
M_{n}=M_{j} n_{j}=N, \\
\tau_{i j} n_{j}+T_{i}^{\prime}=T_{i}, \quad \text { in } \partial \mathscr{D},
\end{gathered}
$$

with $T_{i}^{\prime}:=n_{i}\left(M_{j} n_{j}\right)\left(\Delta_{p} n_{p}\right)-\Delta_{i}\left(M_{j} n_{j}\right)$.

Note. The quantity $M_{l, l}=\operatorname{div} \mathbf{M}$ is a pressure, $T_{i}^{\prime}$ is a force and $M_{n}$ a normal surface double force.

2.2 Constitutive laws. The thermodynamic variables are the absolute temperature $T$, the proportions $\beta_{i}$ 's and the strain tensor $\varepsilon\left(\varepsilon_{i j}(\mathbf{u}):=\left(u_{i, j}+u_{j, i}\right) / 2\right.$, $\mathbf{u}$ being the displacement) and $\operatorname{grad} \operatorname{tr} \varepsilon\left(\operatorname{tr} \varepsilon:=\varepsilon_{j j}\right)$, which are assumed to be the same for the austenite and martensite phases. We suppose that the free energies $\psi_{i}$ of the phases have the following simple and classical expressions:

$$
\begin{aligned}
& \psi_{1}(T, \varepsilon, \operatorname{grad} \operatorname{tr} \varepsilon)=\frac{1}{2} \varepsilon R \varepsilon+\frac{\nu}{2}|\operatorname{grad} \operatorname{tr} \varepsilon|^{2}-\tilde{\alpha}(T) \operatorname{tr} \varepsilon-c_{0} T \log T, \\
& \psi_{2}(T, \varepsilon, \operatorname{grad} \operatorname{tr} \varepsilon)=\frac{1}{2} \varepsilon R \varepsilon+\frac{\nu}{2}|\operatorname{grad} \operatorname{tr} \varepsilon|^{2}+\tilde{\alpha}(T) \operatorname{tr} \varepsilon-c_{0} T \log T, \\
& \psi_{3}(T, \varepsilon, \operatorname{grad} \operatorname{tr} \varepsilon)=\frac{1}{2} \varepsilon R \varepsilon+\frac{\nu}{2}|\operatorname{grad} \operatorname{tr} \varepsilon|^{2}-\frac{l}{T_{0}}\left(T-T_{0}\right)-c_{0} T \log T,
\end{aligned}
$$


where $R$ is the rigidity matrix, $c_{0}$ the heat capacity, $\nu$ a positive coefficient (assumed to be the same for the austenite and martensite phases, still for the sake of simplicity) and $l$ the specific heat of the austenite-martensite phase transition. The function $\tilde{\alpha}(T)$ is proportional to the thermal expansion coefficient. More generally the free energies $\psi_{1}$ and $\psi_{2}$ involve terms linear with respect to $\varepsilon$; here we have chosen the most simple ones. We remark that there is no latent heat in the transition between the two martensitic variants, which are energetically equivalent (the functions $\psi_{1}$ and $\psi_{2}$ are symmetric with respect to the plane $\operatorname{tr} \varepsilon=0$ ). The shape of the function $\tilde{\alpha}$ is shown in Fig. 1; for any temperature $T$ larger than a given temperature $T_{C}\left(T_{C}>T_{0}\right), \tilde{\alpha}(T)$ vanishes.

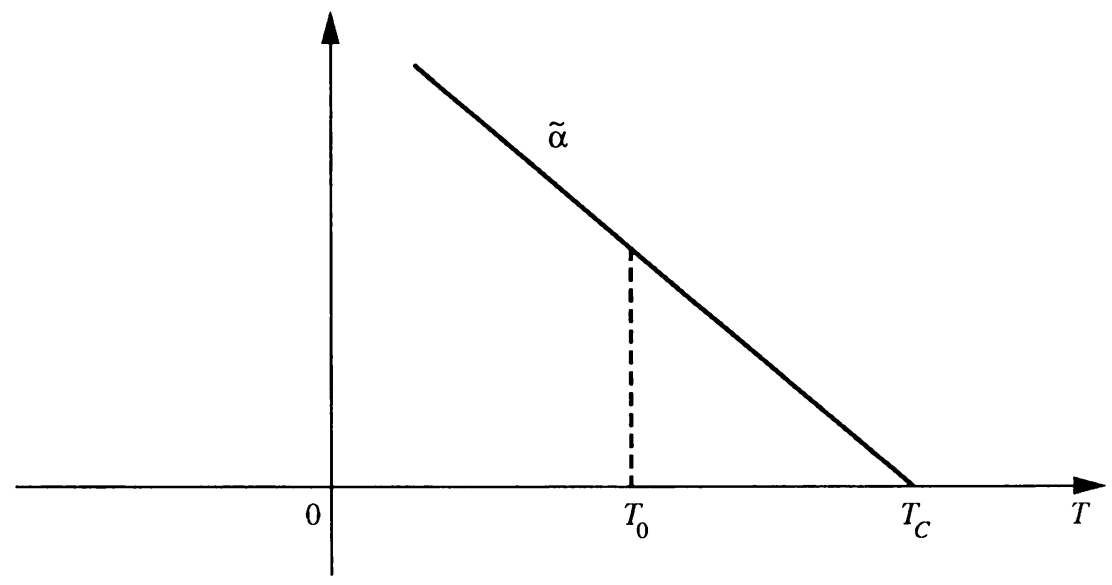

Fig. 1. The graph of the function $\tilde{\alpha}$, proportional to the thermal expansion coefficient.

We also assume that the densities $\rho$ are the same for the three phases. Then the volumetric free energy of the alloy is

$$
\tilde{\psi}\left(T, \varepsilon, \operatorname{grad} \operatorname{tr} \varepsilon, \beta_{1}, \beta_{2}, \beta_{3}\right)=\rho \beta_{i} \psi_{i}+\rho T \widetilde{I}\left(\beta_{1}, \beta_{2}, \beta_{3}\right),
$$

where $\widetilde{I}$ is the indicator function of the convex set

$$
\mathscr{S}:=\left\{\left(\beta_{1}, \beta_{2}, \beta_{3}\right) \mid 0 \leq \beta_{i} \leq 1, \beta_{1}+\beta_{2}+\beta_{3}=1\right\},
$$

namely

$$
\widetilde{I}\left(\beta_{1}, \beta_{2}, \beta_{3}\right)= \begin{cases}0 & \text { if }\left(\beta_{1}, \beta_{2}, \beta_{3}\right) \in \mathscr{S} \\ +\infty & \text { if }\left(\beta_{1}, \beta_{2}, \beta_{3}\right) \notin \mathscr{S} .\end{cases}
$$

The term $\rho T \tilde{I}$ can be understood as a mixture free energy; its only effect is to ensure that the proportions take admissible physical values, i.e., values between 0 and 1 and with sum equal to 1 . We believe that these physical relations are part of the physical properties of mixture and must be included in its free energy. For instance, the fact that the sum of the $\beta_{i}$ 's is 1 expresses that no void can appear and 
that no overlapping can occur. Other examples of such a situation can be found in $[4,9,10]$.

By eliminating $\beta_{3}$, the variables become independent. Then the free energy of the alloy is

$$
\psi\left(T, \varepsilon, \operatorname{grad} \operatorname{tr} \varepsilon, \beta_{1}, \beta_{2}\right)=\rho\left\{\beta_{1}\left(\psi_{1}-\psi_{3}\right)+\beta_{2}\left(\psi_{2}-\psi_{3}\right)+\psi_{3}+T I\left(\beta_{1}, \beta_{2}\right)\right\},
$$

where $I$ is the indicator function of the triangle $\mathscr{T}$ in the plane $\left(\beta_{1}, \beta_{2}\right)$ :

$$
\mathscr{T}=\left\{\left(\beta_{1}, \beta_{2}\right) \mid \beta_{1} \geq 0, \beta_{2} \geq 0, \beta_{1}+\beta_{2} \leq 1\right\} .
$$

By dealing just with the intrinsic dissipation, the Clausius-Duhem inequality derived from the second principle of thermodynamics takes the form

$$
\frac{\partial \psi}{\partial \varepsilon} \dot{\varepsilon}+\frac{\partial \psi}{\partial \operatorname{grad} \operatorname{tr} \varepsilon} \operatorname{grad} \operatorname{tr} \dot{\varepsilon}+\frac{\partial \psi}{\partial \beta_{1}} \dot{\beta}_{1}+\frac{\partial \psi}{\partial \beta_{2}} \dot{\beta}_{2} \leq \sigma \dot{\varepsilon}+\mathbf{M g r a d} \operatorname{tr} \dot{\varepsilon},
$$

where $\sigma$ and $M$ are the actual stresses and the dot denotes the time derivative. The Clausius-Duhem inequality must be satisfied for any choice of the quantities $\dot{\varepsilon}, \operatorname{grad} \operatorname{tr} \dot{\varepsilon}, \dot{\beta}_{1}$, and $\dot{\beta}_{2}$.

We assume that the behaviour is nondissipative or elastic with respect to the mechanical quantities $\varepsilon$ and grad $\operatorname{tr} \varepsilon$ and linearly viscous with respect to the proportions $\beta_{i}$ 's. The constitutive laws are then

$$
\begin{gathered}
\sigma=\frac{\partial \psi}{\partial \varepsilon}=\rho\left(R \varepsilon+\tilde{\alpha}(T)\left(\beta_{2}-\beta_{1}\right) \mathbf{1}\right), \\
\mathbf{M}=\rho \nu \operatorname{grad} \operatorname{tr} \varepsilon \\
k\left(\begin{array}{l}
\dot{\beta}_{1} \\
\dot{\beta}_{2}
\end{array}\right)+\frac{\partial \psi}{\partial \beta} \ni \mathbf{0},
\end{gathered}
$$

where 1 denotes the identity matrix. The latter equation can be written in the form

$$
k\left(\begin{array}{c}
\dot{\beta}_{1} \\
\dot{\beta}_{2}
\end{array}\right)+\mathbf{B}+\rho T \partial I\left(\beta_{1}, \beta_{2}\right) \ni \mathbf{0},
$$

where $\mathbf{B}$ is the smooth part of $\partial \psi / \partial \beta$,

$$
\mathbf{B}=\left(\begin{array}{l}
-\tilde{\alpha}(T) \operatorname{tr} \varepsilon+\frac{l}{T_{0}}\left(T-T_{0}\right) \\
+\tilde{\alpha}(T) \operatorname{tr} \varepsilon+\frac{l}{T_{0}}\left(T-T_{0}\right)
\end{array}\right) .
$$

The viscosity constant $k$ is nonnegative. If $k=0$, the behaviour of the material is nondissipative with respect to $\beta$. It is easy to check that the Clausius-Duhem inequality is then satisfied.

Let us fix the temperature and look at the nondissipative $(k=0)$ constitutive laws. To be clearer, let us consider an extension experiment. As the only nonzero strain $\varepsilon_{11}$ (here replaced by $\varepsilon$, for simplicity) is homogeneous, we have $\mathbf{M}=\mathbf{0}$. We also replace $\sigma_{11}$ with $\sigma$. Now we shall focus our attention onto the relation between $\sigma$ and $\varepsilon$ at different temperatures.

Low temperature $\left(T<T_{0}\right)$. The components of $\mathbf{B}$ are shown in Fig. 3. By (2.6), the vector $-\mathbf{B}$ must be normal to the triangle $\mathscr{T}$ (Fig. 2). We easily obtain

$$
\begin{array}{ll}
\varepsilon=0 \Rightarrow \beta_{1}+\beta_{2}=1, \\
\varepsilon>0 \Rightarrow \beta_{1}=1, & \beta_{2}=0, \\
\varepsilon<0 \Rightarrow \beta_{1}=0, & \beta_{2}=1 .
\end{array}
$$




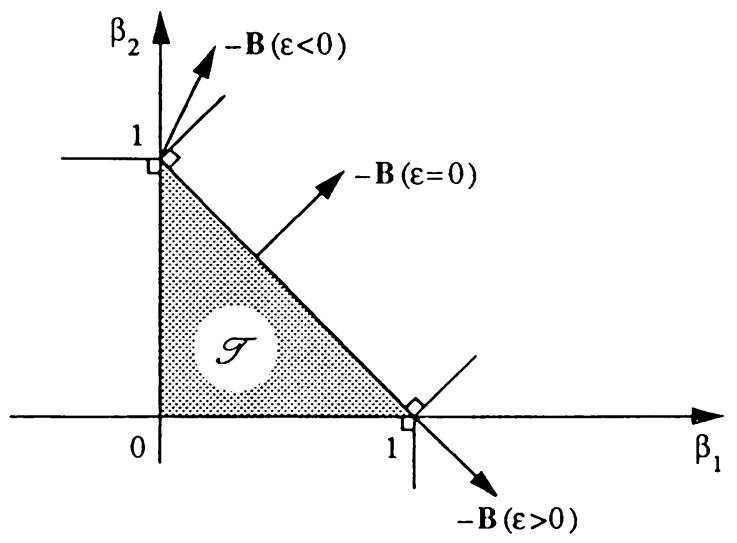

FIG. 2. The triangle $\mathscr{T}$. The vector $-\mathbf{B}$ at a low temperature.

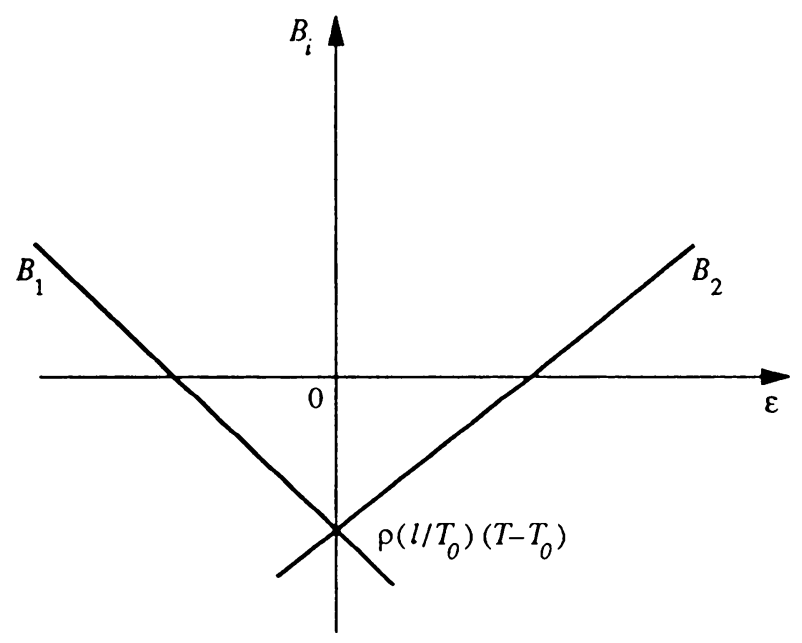

FIG. 3. The component of $\mathbf{B}$ at a low temperature.

Then the relation (2.4) gives the constitutive law shown in Fig. 4, which is quite similar to the experimental law (dotted line). It is easy to check that if the triangle $\mathscr{T}$ is replaced by the curvilinear triangle $\mathscr{T}_{C}$ (Fig. 5) we obtain the experimental law (cf. $[1,7,12,13])$. This means that the only possible mixtures are those represented by the triangle $\mathscr{T}_{C}$ and that the two martensites always coexist. 


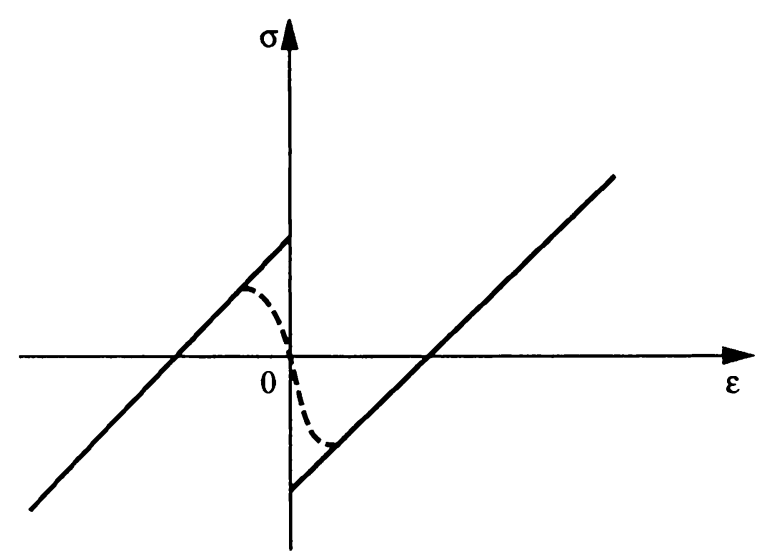

FIG. 4. The mechanical constitutive law at a low temperature. The dotted line represents the experimental law. The first type of the martensite (phase 1) exists for $\varepsilon$ positive, the second one (phase 2) for $\varepsilon$ negative. The two types coexist when $\varepsilon=0$.

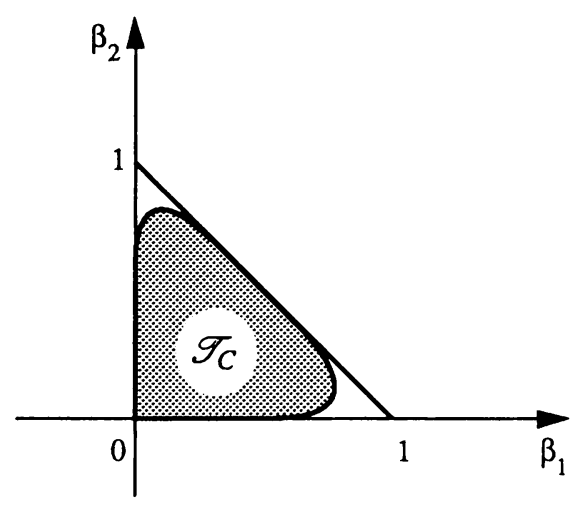

FIG. 5. The triangle $\mathscr{T}_{C}$ gives the experimental behaviour. There is an interaction between the two phases since only certain proportions $\left(\beta_{1}, \beta_{2}\right)$ are possible.

Intermediate temperature $\left(T_{0}<T<T_{C}\right)$. The components of $\mathbf{B}$ are shown in Fig. 6. Relation (2.6) shows that (Fig. 7)

$$
\begin{aligned}
R-\varepsilon_{1}<\varepsilon<\varepsilon_{1} \Rightarrow \beta_{1}=\beta_{2}=0, & \beta_{3}=1 ; \\
\varepsilon=\varepsilon_{1} \Rightarrow \beta_{2}=0, & 0 \leq \beta_{1} \leq 1 ; \\
\varepsilon>\varepsilon_{1} \Rightarrow \beta_{2}=0, & \beta_{1}=1 \\
\varepsilon=-\varepsilon_{1} \Rightarrow \beta_{1}=0, & 0 \leq \beta_{2} \leq 1 ; \\
\varepsilon<-\varepsilon_{1} \Rightarrow \beta_{1}=0, & \beta_{2}=1 .
\end{aligned}
$$




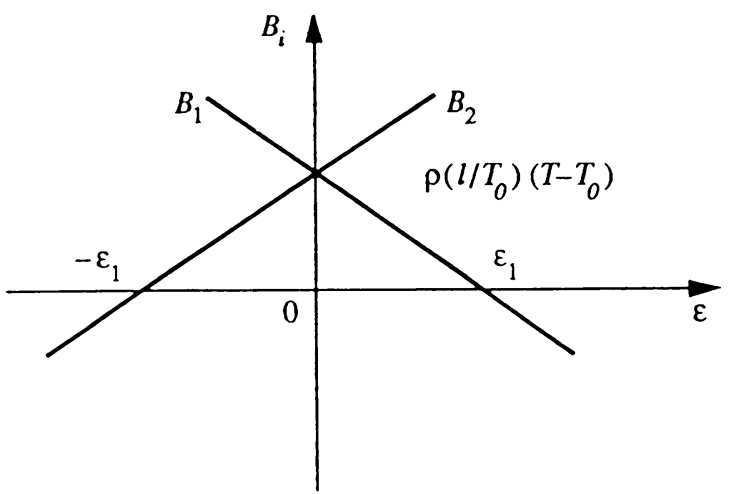

FIG. 6. The components of $\mathbf{B}$ at an intermediate temperature.

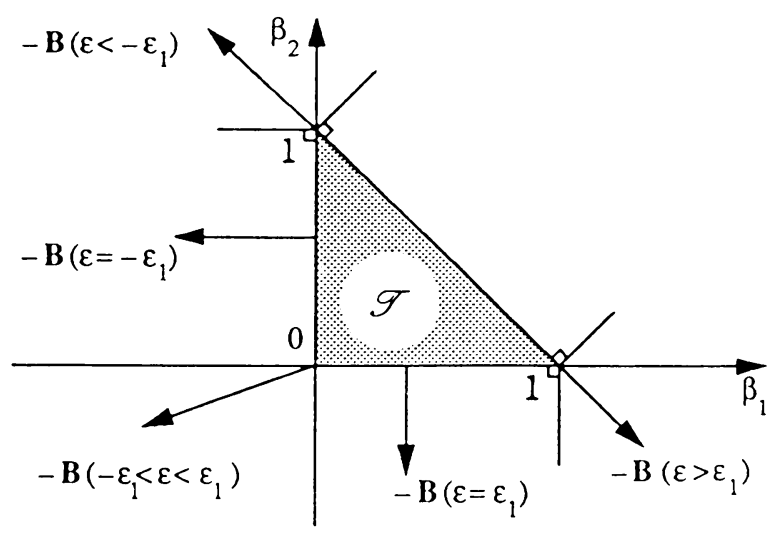

FIG. 7. The vector $-\mathbf{B}$ at an intermediate temperature.

The constitutive law is shown in Fig. 8. Also here it is possible to replace the triangle $\mathscr{T}$ by $\mathscr{T}_{C}$ in order to have a more realistic constitutive law.

High temperature $\left(T>T_{C}\right)$. We have $B_{1}=B_{2}=\rho \frac{l}{T_{0}}\left(T-T_{0}\right)>0$ and by relation (2.6) $\beta_{3}=1$. We then get a classical elastic constitutive law in agreement with experiments (Fig. 9).

Note. When the behaviour is dissipative with respect to the $\beta_{i}$ 's, the evolution is smoother. For instance, if at some fixed temperature the stress $\sigma$ is a given function of time, then the strain $\varepsilon$ evolves in a smoother way.

Note. The nonmonotone constitutive relations outlined in Figs. 4 and 8 are responsible for hysteresis behaviours like those represented in the Figs. 10 and 11.

2.3 Evolution of a shape memory body. In order to describe the evolution of a shape memory material occupying a domain $\Omega$ at each point $x \in \Omega$ and at each instant $t$ of the time interval $[0, \tau]$, we need to know the absolute temperature $T(x, t)$ (or the Celsius temperature $\theta(x, t)=T(x, t)-273$ ), the (small) displacement $\mathbf{u}(x, t)$ and the proportions $\beta_{i}(x, t)$. 


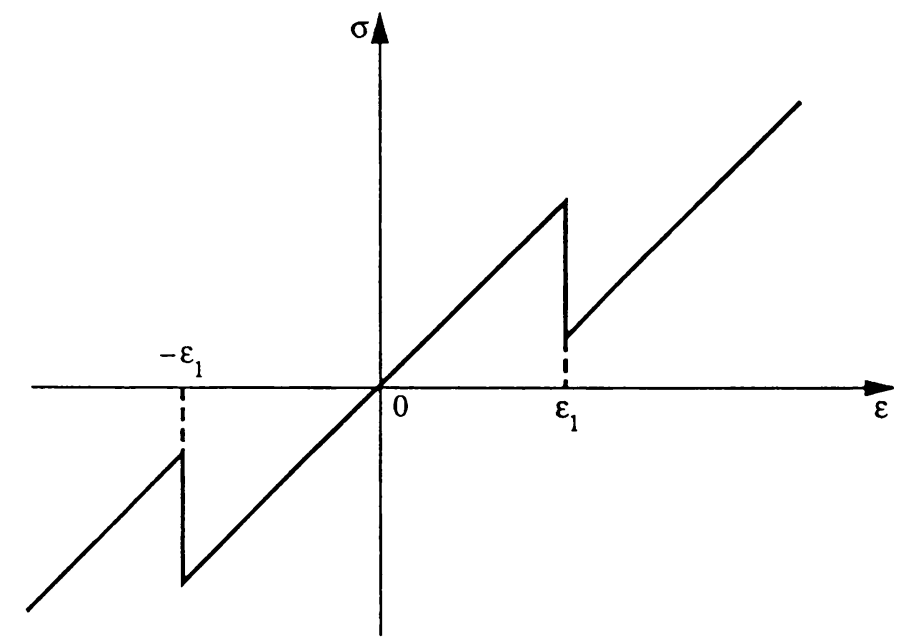

FIG. 8. The constitutive law at an intermediate temperature. The austenite (phase 3 ) exists between $-\varepsilon_{1}$ and $\varepsilon_{1}$. The martensites exist for large deformations (the first type for $\varepsilon>\varepsilon_{1}$, the second one for $\left.\varepsilon<-\varepsilon_{1}\right)$.

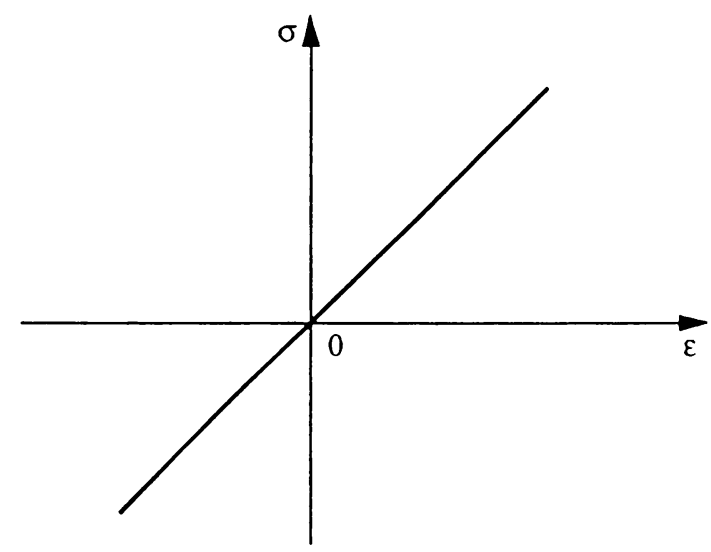

FIG. 9. The constitutive law at a high temperature. Only the austenite (phase 3) exists.

We shall use the energy balance equations

$$
\frac{\partial e}{\partial t}+\operatorname{div} \mathbf{q}=\sigma \dot{\varepsilon}+\mathbf{M g r a d} \operatorname{tr} \dot{\varepsilon},
$$

where $\mathbf{q}$ is the heat flux vector. We neglect the mechanically induced heat sources $\sigma \dot{\varepsilon}+\mathbf{M g r a d} \operatorname{tr} \dot{\varepsilon}$. Moreover in the volumetric internal energy

$$
e=\psi-T \frac{\partial \psi}{\partial T} \simeq \rho\left[c_{0} T-l\left(\beta_{1}+\beta_{2}-1\right)\right],
$$

we consider only the most important terms; namely $c_{0} T$ and especially $l\left(\beta_{1}+\beta_{2}\right)$ which accounts for the austenite-martensite transformation.

We also consider the Fourier conduction law

$$
\mathbf{q}=-h \operatorname{grad} T
$$




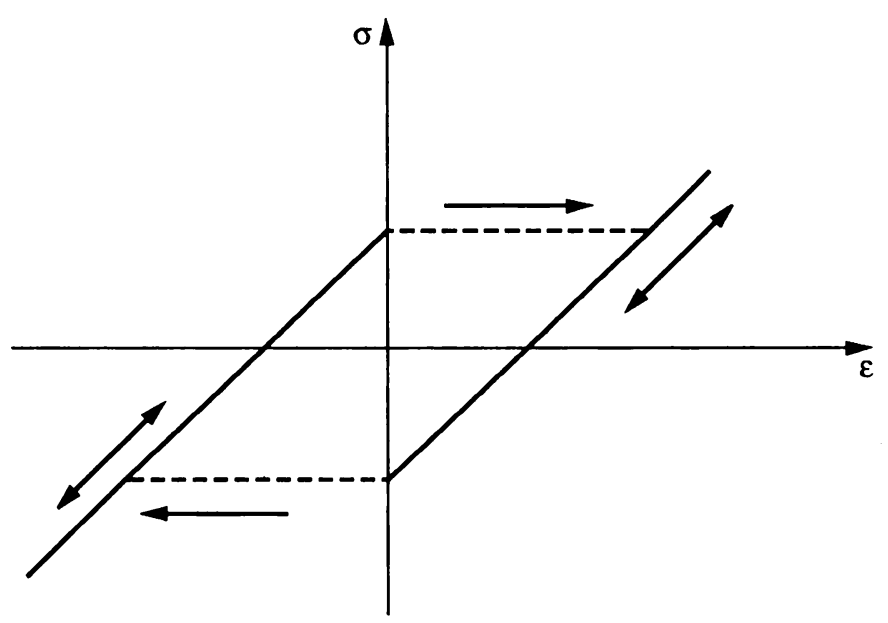

Figure 10

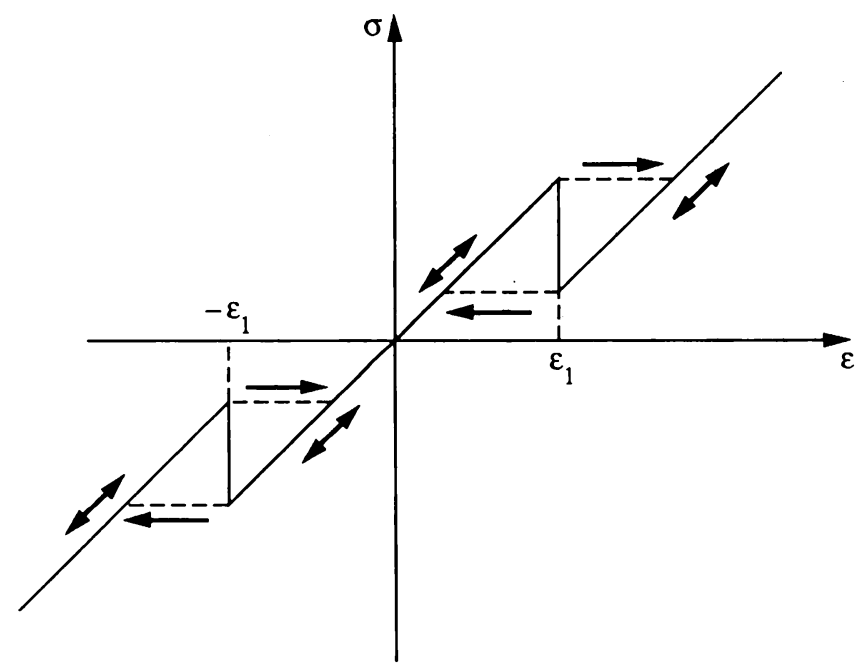

Figure 11

Figs. 10,11. These pictures represent the hysteresis behaviour corresponding to the nonmonotone constitutive relations of Figs. 4, 8. The arrows indicate the directions of the jumps between two monotone branches.

where $h$ is the thermal conductivity.

Let $\alpha(\theta):=\tilde{\alpha}(\theta+273)$. In conclusion, we get the following set of equations:

$$
\left.\rho\left(c_{0} \frac{\partial \theta}{\partial t}-l \frac{\partial}{\partial t}\left(\beta_{1}+\beta_{2}\right)\right)-h \Delta \theta=0 \text { in } Q:=\Omega \times\right] 0, \tau[,
$$


derived from the energy balance;

$$
\begin{gathered}
\operatorname{div}(-\rho \nu \Delta(\operatorname{div} \mathbf{u}) \mathbf{1}+\sigma)=\mathbf{0}, \\
\sigma=\rho\left(R \varepsilon(\mathbf{u})+\alpha(\theta)\left(\beta_{2}-\beta_{1}\right) \mathbf{1}\right), \quad \text { in } Q,
\end{gathered}
$$

derived from the equilibrium equations and from the mechanical constitutive laws (2.1)-(2.5) (where we take $\mathbf{f}=\mathbf{0}$ );

$$
k \frac{\partial}{\partial t}\left(\begin{array}{l}
\beta_{1} \\
\beta_{2}
\end{array}\right)+\partial I\left(\beta_{1}, \beta_{2}\right) \ni\left(\begin{array}{c}
1 \\
-1
\end{array}\right) \alpha(\theta) \operatorname{div} \mathbf{u}-\frac{l}{T_{0}}\left(\begin{array}{l}
1 \\
1
\end{array}\right)\left(\theta-\theta^{*}\right) \text { in } Q,
$$

derived from the constitutive law $(2.6)$ for the $\beta_{i}$ 's (note that $\rho T \partial I=\partial I, \theta^{*}:=$ $\left.T_{0}-273\right)$.

Finally we need suitable boundary and initial conditions. For instance, we choose as boundary conditions

$$
\begin{gathered}
\left.\mathbf{u}=\mathbf{0} \quad \text { on } \Sigma_{0}:=\partial \Omega_{0} \times\right] 0, \tau[, \\
\left.(-\rho \nu \Delta(\operatorname{div} \mathbf{u}) \mathbf{1}+\sigma) \mathbf{n}=\mathbf{g} \quad \text { on } \Sigma_{1}:=\partial \Omega_{1} \times\right] 0, \tau[,
\end{gathered}
$$

where $\partial \Omega_{0} \cup \partial \Omega_{1}=\partial \Omega, \partial \Omega_{0} \cap \partial \Omega_{1}=\varnothing$, the surface measure of $\partial \Omega_{0}$ is positive and $\mathbf{g}$ is the external traction applied to $\partial \Omega_{1}$ (cf. (2.3)). Assuming that no double force is applied, we take (cf. (2.2))

$$
\left.\frac{\partial}{\partial n}(\operatorname{div} \mathbf{u})=0 \quad \text { on } \Sigma:=\partial \Omega \times\right] 0, \tau[\text {. }
$$

Finally, let

$$
h \frac{\partial \theta}{\partial n}+\bar{a}(\theta-\bar{\theta})=0 \text { on } \Sigma,
$$

i.e., the heat flux is proportional to the difference of temperature between the interior $(\theta)$ and the exterior $(\bar{\theta})$ of the system ( $\bar{a}$ being a positive parameter). As initial conditions, we consider

$$
\theta(x, 0)=\theta^{0}(x), \quad \beta_{1}(x, 0)=\beta_{1}^{0}(x), \quad \beta_{2}(x, 0)=\beta_{2}^{0}(x) \quad \text { for } x \in \Omega .
$$

Then the unknowns of the problem are the functions $\theta(x, t), \mathbf{u}(x, t), \beta_{1}(x, t)$, $\beta_{2}(x, t)$. The data are $c_{0}, 1, \rho, \nu, \bar{a}, \alpha(\theta), h, T_{0}, T_{c}, k, \mathbf{g}, \bar{\theta}, \theta^{0}, \beta_{1}^{0}, \beta_{2}^{0}$, and the rigidity matrix $R$. As it is usual in elasticity we assume the material to be homogeneous and isotropic:

$$
\rho R \varepsilon(\mathbf{u}):=\lambda \operatorname{div} \mathbf{u} \mathbf{1}+2 \mu \varepsilon(\mathbf{u}),
$$

where $\lambda$ and $\mu$ are the Lamé constants. Finally the equations of the problem are the relations $(2.7)-(2.13)$.

3. Variational formulation and theorem of existence and uniqueness. First we recall the mathematical problem. Let $\Omega$ be a bounded domain in $\mathbf{R}^{3}$ with a boundary $\partial \Omega$ of class $C^{1,1}$ in the sense of [15], and let $\left(\Gamma_{0}, \Gamma_{1}\right)$ be a partition of $\partial \Omega$ into two measurable subsets such that $\Gamma_{0}$ has positive surface measure. We denote by $\mathbf{n}$ the outward normal unit vector to $\partial \Omega$. We fix any positive time $\tau$ and set

$$
Q:=\Omega \times] 0, \tau[, \quad \Sigma:=\partial \Omega \times] 0, \tau\left[, \quad \Sigma_{j}:=\Gamma_{j} \times\right] 0, \tau[, \quad j=0,1 .
$$


Let $\alpha: \mathbf{R} \rightarrow \mathbf{R}$ be a bounded and Lipschitz continuous function, and let $\widetilde{K}$ be a nonempty closed convex subset of $R^{2}$ such that

$$
\widetilde{K} \subseteq\left\{\left(\beta_{1}, \beta_{2}\right) \in \mathbf{R}^{2}: \beta_{1} \geq 0, \beta_{2} \geq 0, \beta_{1}+\beta_{2} \leq 1\right\} .
$$

The physical constants are as defined above. With standard notations $\nabla, \Delta$ and div will denote the gradient, Laplacian and divergence operators, respectively, and $\sigma$ and $\varepsilon$ the stress and strain tensors. Given

$$
\text { g: } \Sigma_{1} \rightarrow \mathbf{R}^{3}, \quad \bar{\theta}: \Sigma \rightarrow \mathbf{R}, \quad \theta^{0}, \beta_{1}^{0}, \beta_{2}^{0}: \Omega \rightarrow \mathbf{R},
$$

we look for functions $\mathbf{u}=\left(u_{1}, u_{2}, u_{3}\right): Q \rightarrow \mathbf{R}^{3}, \theta, \beta_{1}, \beta_{2}: Q \rightarrow \mathbf{R}$ such that, setting

$$
\begin{gathered}
\varepsilon_{i j}(\mathbf{u}):=\frac{1}{2}\left(\frac{\partial u_{i}}{\partial x_{j}}+\frac{\partial u_{j}}{\partial x_{i}}\right) \quad \text { in } Q, i, j=1,2,3, \\
\sigma:=\lambda(\operatorname{div} \mathbf{u}) \mathbf{1}+2 \mu \varepsilon(\mathbf{u})+\alpha(\theta)\left(\beta_{2}-\beta_{1}\right) \mathbf{1} \text { in } Q,
\end{gathered}
$$

and assuming for simplicity $\rho=1$, the following equations hold

$$
\begin{gathered}
c_{0} \frac{\partial \theta}{\theta t}-l \frac{\partial}{\partial t}\left(\beta_{1}+\beta_{2}\right)-h \Delta \theta=0 \quad \text { in } Q, \\
\operatorname{div}(-\nu \Delta(\operatorname{div} \mathbf{u}) \mathbf{1}+\sigma)=\mathbf{0} \quad \text { in } Q, \\
k \frac{\partial}{\partial t}\left(\begin{array}{c}
\beta_{1} \\
\beta_{2}
\end{array}\right)+\partial I_{\widetilde{K}}\left(\beta_{1}, \beta_{2}\right) \ni\left(\begin{array}{c}
1 \\
-1
\end{array}\right) \alpha(\theta) \operatorname{div} \mathbf{u}-\frac{l}{T_{0}}\left(\begin{array}{l}
1 \\
1
\end{array}\right)\left(\theta-\theta^{*}\right) \quad \text { in } Q, \\
\mathbf{u}=\mathbf{0} \quad \text { on } \Sigma_{0}, \\
(-\nu \Delta(\operatorname{div} \mathbf{u}) \mathbf{1}+\sigma) \cdot \mathbf{n}=\mathbf{g} \quad \text { on } \Sigma_{1}, \\
\frac{\partial}{\partial n}(\operatorname{div} \mathbf{u})=0 \quad \text { on } \Sigma, \\
h \frac{\partial \theta}{\partial n}+\bar{a}(\theta-\bar{\theta})=0 \quad \text { on } \Sigma, \\
\theta(\cdot, 0)=\theta^{0}, \quad \beta_{1}(\cdot, 0)=\beta_{1}^{0}, \quad \beta_{2}(\cdot, 0)=\beta_{2}^{0} \quad \text { in } \Omega .
\end{gathered}
$$

Here $\partial I_{\widetilde{K}}$ denotes the subdifferential of $I_{\widetilde{K}}$, indicator function of the closed convex set $\widetilde{K}$. We set

$$
\chi_{1}:=\frac{1}{\sqrt{2}}\left(\beta_{1}+\beta_{2}\right), \quad \chi_{2}:=\frac{1}{\sqrt{2}}\left(\beta_{2}-\beta_{1}\right) .
$$

Then $\left(\beta_{1}, \beta_{2}\right) \in \widetilde{K}$ if and only if

$$
\left(\chi_{1}, \chi_{2}\right) \in K:=\left\{\left(\chi_{1}, \chi_{2}\right) \in \mathbf{R}^{2}: \frac{\chi_{1}-\chi_{2}}{\sqrt{2}}, \frac{\chi_{1}+\chi_{2}}{\sqrt{2}} \in \tilde{K}\right\},
$$

and also $K$ is a nonempty closed convex set included in

$$
\left\{\left(\chi_{1}, \chi_{2}\right) \in \mathbf{R}^{2}:\left|\chi_{2}\right| \leq \chi_{1} \leq \frac{\sqrt{2}}{2}\right\} .
$$

Thus (3.5) can be rewritten in the equivalent form

$$
k \frac{\partial}{\partial t}\left(\begin{array}{l}
\chi_{1} \\
\chi_{2}
\end{array}\right)+\partial I_{K}\left(\chi_{1}, \chi_{2}\right) \ni\left(\begin{array}{c}
-\frac{\sqrt{2} l}{T_{0}}\left(\theta-\theta^{*}\right) \\
-\sqrt{2} \alpha(\theta) \operatorname{div} \mathbf{u}
\end{array}\right) \quad \text { in } Q
$$


and in (3.2), (3.3), (3.10) $\beta_{1}$ and $\beta_{2}$ can be replaced respectively by $\frac{1}{\sqrt{2}}\left(\chi_{1}-\chi_{2}\right)$, $\frac{1}{\sqrt{2}}\left(\chi_{1}, \chi_{2}\right)$. In order to give a precise formulation of the previous problem, we introduce the closed convex subset of $\left(L^{2}(\Omega)\right)^{2}$

$$
K:=\left\{\left(\chi_{1}, \chi_{2}\right) \in\left(L^{2}(\Omega)\right)^{2}:\left(\chi_{1}, \chi_{2}\right) \in K \text { a.e. in } \Omega\right\},
$$

and the space

$$
V:=\left\{\mathbf{u} \in\left(H^{1}(\Omega)\right)^{3}: \mathbf{u}=\mathbf{0} \text { on } \Gamma_{0}, \operatorname{div} \mathbf{u} \in H^{1}(\Omega)\right\} .
$$

$V$ is a Hilbert space with corresponding norm

$$
\|\mathbf{u}\|_{V}:=\left\{\sum_{i=1}^{3}\left(\int_{\Omega}\left(\frac{\partial}{\partial x_{i}}(\operatorname{div} \mathbf{u})\right)^{2} d x+\sum_{j=1}^{3} \int_{\Omega}\left(\frac{\partial u_{j}}{\partial x_{i}}\right)^{2} d x\right)\right\}^{1 / 2} .
$$

We also set for any $\mathbf{u}, \mathbf{v} \in V$

$$
\begin{aligned}
a(\mathbf{u}, \mathbf{v}):= & \sum_{i=1}^{3} \nu \int_{\Omega} \frac{\partial}{\partial x_{i}}(\operatorname{div} \mathbf{u}) \frac{\partial}{\partial x_{i}}(\operatorname{div} \mathbf{v}) d x \\
& +\lambda \int_{\Omega} \varepsilon_{i i}(\mathbf{u}) \varepsilon_{j j}(\mathbf{v}) d x+\sum_{i, j=1}^{3} 2 \mu \int_{\Omega} \varepsilon_{i j}(\mathbf{u}) \varepsilon_{i j}(\mathbf{v}) d x
\end{aligned}
$$

$a(\cdot, \cdot)$ is a bilinear continuous form on $V \times V$, and the inequalities $\nu>0, \mu>0$, $3 \lambda+2 \mu>0$ yield the existence of a constant $C>0$ such that

$$
a(\mathbf{u}, \mathbf{u}) \geq C\|\mathbf{u}\|_{V}^{2} \quad \forall \mathbf{u} \in V .
$$

Henceforth we shall denote by $\langle\cdot, \cdot\rangle$ the duality pairing between $\left(H^{1}(\Omega)\right)^{\prime}$ and $H^{1}(\Omega)$. We assume that

$$
\begin{gathered}
\alpha \in W^{1, \infty}(\mathbf{R}) ; \\
\theta^{0} \in L^{2}(\Omega) ; \quad\left(\chi_{1}^{0}, \chi_{2}^{0}\right) \in K ; \\
g \in L^{\infty}\left(0, \tau ;\left(L^{2}\left(\Gamma_{1}\right)\right)^{3}\right) ; \quad \bar{\theta} \in L^{2}(\Sigma) .
\end{gathered}
$$

A precise formulation of the problem is as follows.

Problem (P). Find $\theta \in L^{2}\left(0, \tau ; H^{1}(\Omega)\right) \cap H^{1}\left(0, \tau ;\left(H^{1}(\Omega)\right)^{\prime}\right), \mathbf{u} \in L^{\infty}(0, \tau ; V), \chi_{1}, \chi_{2}$ $\in H^{1}\left(0, \tau ; L^{2}(\Omega)\right)$ satisfying

$$
\left(\chi_{1}, \chi_{2}\right) \in K
$$

$\left\langle\frac{d}{d t}\left(c_{0} \theta-\sqrt{2} l \chi_{1}\right), \varphi\right\rangle+h \int_{\Omega} \nabla \theta \nabla \varphi d x+\bar{a} \int_{\partial \Omega}(\theta-\bar{\theta}) \varphi d \Gamma=0 \quad \forall \varphi \in H^{1}(\Omega)$,

$$
\begin{gathered}
a(\mathbf{u}, \mathbf{v})+\int_{\Omega} \sqrt{2} \alpha(\theta) \chi_{2} \operatorname{div} \mathbf{v} d x=\int_{\Gamma_{1}} \mathbf{g} \cdot \mathbf{v} d \Gamma \quad \forall \mathbf{v} \in V \\
\int_{\Omega}\left\{k \sum_{i=1}^{2}\left[\frac{\partial \chi_{i}}{\partial t}\left(\chi_{i}-\gamma_{i}\right)\right]+\frac{\sqrt{2} l}{T_{0}}\left(\theta-\theta^{*}\right)\left(\chi_{1}-\gamma_{1}\right)+\sqrt{2} \alpha(\theta) \operatorname{div} \mathbf{u}\left(\chi_{2}-\gamma_{2}\right)\right\} d x \leq 0 \\
\forall\left(\gamma_{1}, \gamma_{2}\right) \in K
\end{gathered}
$$


a.e. in $] 0, \tau[$, and such that

$$
\begin{gathered}
\left.\theta\right|_{t=0}=\theta^{0} \quad \text { in }\left(H^{1}(\Omega)\right)^{\prime}, \\
\left.\left(\chi_{1}, \chi_{2}\right)\right|_{t=0}=\left(\chi_{1}^{0}, \chi_{2}^{0}\right) \quad \text { a.e. in } \Omega .
\end{gathered}
$$

In order to solve this problem, first we present some auxiliary lemmata.

LemmA 1. For any $\theta, \chi_{2} \in L^{2}(Q)$, such that $\left|\chi_{2}\right| \leq \frac{\sqrt{2}}{2}$ a.e. in $Q$ (cf. the definition of $K)$, there exists one and only one $\mathbf{u}=B\left(\theta, \chi_{2}\right) \in L^{\infty}(0, \tau ; V)$ satisfying (3.18) a.e. in $] 0, \tau[$. Moreover

$$
\|\operatorname{div} \mathbf{u}\|_{L^{\infty}(Q)} \leq C_{1}\|\operatorname{div} \mathbf{u}\|_{L^{\infty}\left(0, \tau ; H^{2}(\Omega)\right)} \leq C_{2},
$$

where $C_{1}$ is a constant given by the Sobolev inclusion $H^{2}(\Omega) \hookrightarrow L^{\infty}(\Omega)$ and $C_{2}$ is a constant depending only on $\Omega, C_{1}, C,\|\mathbf{g}\|_{\left.L^{\infty}\left(0, \tau ; L^{2}\left(\Gamma_{1}\right)\right)^{3}\right)},\|\alpha\|_{L^{\infty}(\mathbf{R})}$.

Proof. The existence and the uniqueness of $\mathbf{u}$ follow directly from the Lax-Milgram Theorem (cf., e.g., [15]), since the form $a(\cdot, \cdot)$ is $V$-elliptic (see (3.13)) and

$$
\int_{\Gamma_{1}} \mathbf{g} \cdot \mathbf{v} d \Gamma-\int_{\Omega} \sqrt{2} \alpha(\theta) \chi_{2} \operatorname{div} \mathbf{v} d x
$$

is a linear and continuous operator on $V$. Moreover

$$
\|\mathbf{u}\|_{L^{\infty}(0, \tau ; V)} \leq C_{3}
$$

where the constant $C_{3}$ has the same dependence of $C_{2}$, and the solution $\mathbf{u}$ verifies (cf. (3.1), (3.2), (3.4))

$$
\mu \Delta u_{i}+\frac{\partial}{\partial x_{i}}\left[(\lambda+\mu) \operatorname{div} \mathbf{u}-\nu \Delta(\operatorname{div} \mathbf{u})+\sqrt{2} \alpha(\theta) \chi_{2}\right]=0, \quad i=1,2,3,
$$

in $\mathscr{D}^{\prime}(\Omega)$, for a.e. $\left.t \in\right] 0, \tau[$ and the boundary conditions (3.6), (3.7), (3.8) in a suitable sense. Owing to (3.24), from (3.25) it follows that

$$
\left\|(\lambda+u) \operatorname{div} \mathbf{u}-\nu \Delta(\operatorname{div} \mathbf{u})+\alpha(\theta) \sqrt{2} \chi_{2}\right\|_{L^{\infty}\left(0, \tau ; L^{2}(\Omega)\right)} \leq C_{4},
$$

where $C_{4}$ is a constant depending only on $\Omega$ and $C_{3}$. Thus $\Delta(\operatorname{div} \mathbf{u})$ is bounded in $L^{\infty}\left(0, \tau ; L^{2}(\Omega)\right)$ and $\operatorname{div} \mathbf{u}$ satisfies the homogeneous boundary condition (3.8). The estimate (3.22) follows from the regularity results for elliptic boundary value problems (see, for instance, [15]).

The next two lemmata are an easy consequence of the theory of parabolic variational inequalities (see, e.g., [6]).

LemmA 2. Given $\theta \in L^{2}(\Omega), \mathbf{u} \in L^{2}\left(0, \tau ;\left(H^{1}(\Omega)\right)^{3}\right)$, there exists one and only one pair $\left(\chi_{1}, \chi_{2}\right)=D(\mathbf{u}, \theta) \in H^{1}\left(0, \tau ;\left(L^{2}(\Omega)\right)^{2}\right)$ satisfying (3.16), (3.19) a.e. in ]0, $\tau$ [ and (3.21).

Lemma 3. Given $\chi_{1} \in H^{1}\left(0, \tau ; L^{2}(\Omega)\right)$, there exists a unique $\theta=E\left(\chi_{1}\right) \in$ $L^{2}\left(0, \tau ; H^{1}(\Omega)\right) \cap H^{1}\left(0, \tau ;\left(H^{1}(\Omega)\right)^{\prime}\right) \cap C^{0}\left([0, \tau] ; L^{2}(\Omega)\right)$, verifying (3.17) a.e. in ]0, $\tau\left[\right.$ and (3.20). Moreover, if $\theta^{0} \in H^{1}(\Omega)$ and $\bar{\theta} \in W^{1,1}\left(0, \tau ; L^{2}(\partial \Omega)\right)$, then $\theta \in$ $H^{1}\left(0, \tau ; L^{2}(\Omega)\right) \cap L^{\infty}\left(0, \tau ; H^{1}(\Omega)\right)$.

Now, we show the existence and uniqueness theorem. 
Theorem 1. Problem (P) has one and only one solution. Moreover, if $\theta^{0} \in H^{1}(\Omega)$ and $\bar{\theta} \in W^{1,1}\left(0, \tau ; L^{2}(\partial \Omega)\right)$, then $\theta \in H^{1}\left(0, \tau ; L^{2}(\Omega)\right) \cap L^{\infty}\left(0, \tau ; H^{1}(\Omega)\right)$.

Proof. First we outline our procedure. For any $\hat{\theta} \in L^{2}(Q), \hat{\chi}_{2} \in L^{\infty}(Q)$ such that $\left\|\hat{\chi}_{2}\right\|_{L^{\infty}(Q)} \leq \frac{\sqrt{2}}{2}$ (cf. the definition of $K$ ), we replace $\theta$ and $\chi_{2}$ with $\hat{\theta}$ and $\hat{\chi}_{2}$ in (3.18); this determines $\mathbf{u}=B\left(\hat{\theta}, \hat{\chi}_{2}\right)$ (see Lemma 1 ). Then, using $\mathbf{u}$ and $\hat{\theta}$ (instead of $\theta$ ), we get $\left(\chi_{1}, \chi_{2}\right)=D(\mathbf{u}, \hat{\theta})$ from (3.16), (3.19) and (3.21) (cf. Lemma 2$)$; finally we find the solution $\theta=E\left(\chi_{1}\right)$ of (3.17), (3.20) (cf. Lemma 3). Setting $\left.(\bar{t} \in] 0, \tau\right]$ to be specified later)

$$
X:=\left\{\left(\varphi, \gamma_{2}\right) \in\left(L^{2}(\Omega \times] 0, \bar{t}[)\right)^{2}:\left|\gamma_{2}(x, t)\right| \leq \frac{\sqrt{2}}{2} \text { a.e. in } \Omega \times\right] 0, \bar{t}[\},
$$

the above construction defines an operator

$$
A: X \rightarrow X, \quad A\left(\hat{\theta}, \hat{\chi}_{2}\right)=\left(\theta, \chi_{2}\right) .
$$

We want to show that for sufficiently small $\bar{t}, A$ is a contraction mapping on $X$; then $A$ will have a unique fixed point and, repeating this procedure step by step in time (notice that each time interval can be assumed of length $\bar{t}$ ), we shall get the result.

Now we fix any $\left(\hat{\theta}_{i}, \hat{\chi}_{2 i}\right) \in X$ and set $\mathbf{u}_{i}:=B\left(\hat{\theta}_{i}, \hat{\chi}_{2 i}\right),\left(\chi_{1 i}, \chi_{2 i}\right):=D\left(\mathbf{u}_{i}, \hat{\theta}_{i}\right)$ and $\theta_{i}:=E\left(\chi_{1 i}\right), i=1,2$. Then we have $\left(\theta_{i}, \chi_{2 i}\right)=A\left(\hat{\theta}_{i}, \hat{\chi}_{2 i}\right)$.

We write (3.18) with $\hat{\theta}_{i}, \hat{\chi}_{2 i}$ instead of $\theta, \chi_{2}$ for $i=1,2$, take their difference, choose $\mathbf{v}=\mathbf{u}_{1}-\mathbf{u}_{2}(\in V$ for a.e. $t \in] 0, \bar{t}[)$ and integrate in time. With the help of (3.13), this yields for any $t \in[0, \bar{t}]$

$$
\begin{aligned}
\int_{0}^{t}\left\|\mathbf{u}_{1}(\cdot, s)-\mathbf{u}_{2}(\cdot, s)\right\|_{V}^{2} d s \leq \frac{\sqrt{2}}{C} & \left\{\int_{0}^{t} d s \int_{\Omega}\left|\alpha\left(\hat{\theta}_{1}\right)-\alpha\left(\hat{\theta}_{2}\right)\right|\left|\hat{\chi}_{21}\right|\left|\operatorname{div}\left(\mathbf{u}_{1}-\mathbf{u}_{2}\right)\right| d x\right. \\
& \left.+\int_{0}^{t} d s \int_{\Omega}\left|\hat{\chi}_{21}-\hat{\chi}_{22}\right|\left|\alpha\left(\hat{\theta}_{2}\right)\right|\left|\operatorname{div}\left(\mathbf{u}_{1}-\mathbf{u}_{2}\right)\right| d x\right\} .
\end{aligned}
$$

Now, taking into account (3.14), (3.27) and utilizing the elementary inequality

$$
2 a_{1} a_{2} \leq \delta a_{1}^{2}+\frac{1}{\delta} a_{2}^{2} \quad \forall \delta>0, a_{1}, a_{2} \in \mathbf{R},
$$

from (3.29) we obtain

$$
\left\|\mathbf{u}_{1}-\mathbf{u}_{2}\right\|_{L^{2}(0, \bar{t} ; V)}^{2} \leq C_{5}\left\{\left\|\hat{\theta}_{1}-\hat{\theta}_{2}\right\|_{L^{2}(\Omega \times] 0, \bar{t}[)}^{2}+\left\|\hat{\chi}_{21}-\hat{\chi}_{22}\right\|_{\left.L^{2}(\Omega \times] 0, \bar{t}\right)}^{2}\right\},
$$

where, for instance, $C_{5}=\left(48 / C^{2}\right)\|\alpha\|_{W^{1, \infty}(\mathbf{R})}^{2}$.

Then we replace $\theta$, u with $\hat{\theta}_{j}, \mathbf{u}_{j}, j=1,2$ in (3.19) and write (3.19) for $\chi_{i j}$, $j=1,2$ taking, respectively, $\gamma_{i}=\chi_{i 2}$ and $\gamma_{i}=\chi_{i 1}$ for $i=1,2$. Then we sum them and integrate in time; notice that both $\left(\chi_{1 j}, \chi_{2 j}\right), j=1,2$, verify (3.16) and (3.21). 
For any $t \in[0, \bar{t}]$ we get

$$
\begin{aligned}
\frac{1}{2} \sum_{i=1}^{2}\left\|\chi_{i 1}(\cdot, t)-\chi_{i 2}(\cdot, t)\right\|_{L^{2}(\Omega)}^{2} & \\
\leq \frac{\sqrt{2}}{k} \max \left[1, \frac{l}{T_{0}}\right]\{ & \int_{0}^{t} d s \int_{\Omega}\left|\hat{\theta}_{1}-\hat{\theta}_{2}\right|\left|\chi_{11}-\chi_{12}\right| d x \\
& +\left\|\operatorname{div} \mathbf{u}_{1}\right\|_{L^{\infty}(\Omega \times], 0, t[)} \int_{0}^{t} d s \int_{\Omega}\left|\alpha\left(\hat{\theta}_{1}\right)-\alpha\left(\hat{\theta}_{2}\right)\right|\left|\chi_{21}-\chi_{22}\right| d x \\
& \left.\left.+\|\alpha\|_{L^{\infty}(\mathbf{R})} \int_{0}^{t} d s \int_{\Omega}\left|\operatorname{div}\left(\mathbf{u}_{1}-\mathbf{u}_{2}\right)\right|\left|\chi_{21}-\chi_{22}\right| d x\right\} ; \quad 3.32\right)
\end{aligned}
$$

hence from Lemma 1, (3.14), (3.32) and with the help of Gronwall Lemma (see, for instance, [5], Appendix) we obtain

$$
\sum_{i=1}^{2}\left\|\chi_{i 1}(\cdot, t)-\chi_{i 2}(\cdot, t)\right\|_{L^{2}(\Omega)} \leq C_{6} \int_{0}^{t}\left\{\left\|\theta_{1}-\theta_{2}\right\|_{L^{2}(\Omega)}+\left\|\operatorname{div}\left(\mathbf{u}_{1}-\mathbf{u}_{2}\right)\right\|_{L^{2}(\Omega)}\right\} d s
$$

$$
\forall t \in[0, \bar{t}],
$$

where, for example, $C_{6}=\frac{2}{k} \max \left[1, \frac{l}{T_{0}}\right]\left\{1+\left(C_{2}+1\right)\|\alpha\|_{W^{1, \infty}(\mathbf{R})}\right\}$. Now from (3.33) and applying Hölder inequality in time, it follows that

$$
\begin{array}{r}
\sum_{i=1}^{2}\left\|\chi_{i 1}(\cdot, t)-\chi_{i 2}(\cdot, t)\right\|_{L^{2}(\Omega)} \leq \sqrt{3} C_{6} t^{1 / 2}\left\{\left\|\hat{\theta}_{1}-\hat{\theta}_{2}\right\|_{L^{2}(\Omega \times] 0, \bar{t}[)}+\left\|\mathbf{u}_{1}-\mathbf{u}_{2}\right\|_{L^{2}(0, \bar{t} ; V)}\right\} \\
\forall t \in[0, \bar{t}] .
\end{array}
$$

Then in (3.17) we replace $\chi_{1}$ with $\chi_{1 j}, j=1,2$ and write (3.17) for $j=1,2$, take their difference, integrate it in time from 0 to $t$. Taking into account that both $\left(\theta_{1}, \chi_{11}\right)$ and $\left(\theta_{2}, \chi_{12}\right)$ satisfy $(3.20)$ and $(3.21)$, we obtain for any $t \in[0, \bar{t}]$

$$
\begin{aligned}
c_{0} \int_{\Omega}\left(\theta_{1}(\cdot, t)\right. & \left.-\theta_{2}(\cdot, t)\right) \varphi d x+h \int_{\Omega} \nabla \int_{0}^{t}\left(\theta_{1}-\theta_{2}\right) d s \nabla \varphi d x \\
& +\bar{a} \int_{\partial \Omega} \int_{0}^{t}\left(\theta_{1}-\theta_{2}\right) d s \varphi d \Gamma=\sqrt{2} l \int_{\Omega}\left(\chi_{11}(\cdot, t)-\chi_{12}(\cdot, t)\right) \varphi d x .
\end{aligned}
$$

Taking $\varphi=\theta_{1}-\theta_{2}$ and integrating once more in time, with the help of (3.30) we easily get

$$
\left\|\theta_{1}-\theta_{2}\right\|_{L^{2}(\Omega \times] 0, \bar{t}[)}^{2} \leq C_{7}\left\|\chi_{11}-\chi_{12}\right\|_{L^{2}(\Omega \times] 0, \bar{t}[)}^{2}
$$

where, for instance, $C_{7}=8 l^{2} / c_{0}^{2}$. Finally (3.31), (3.34), (3.36) yield

$$
\begin{aligned}
\| \theta_{1} & \theta_{2}\left\|_{L^{2}(\Omega \times] 0, \bar{t}[)}^{2}+\right\| \chi_{21}-\chi_{22} \|_{L^{2}(\Omega \times] 0, \bar{l}[)}^{2} \\
& \leq \max \left\{1, C_{7}\right\} \sum_{i=1}^{2}\left\|\chi_{i 1}-\chi_{i 2}\right\|_{L^{2}(\Omega \times] 0, \bar{t}[)}^{2} \\
& \leq \bar{t} \max \left\{1, C_{7}\right\} \sup _{0 \leq t \leq \bar{t}} \sum_{i=1}^{2}\left\|\chi_{i 1}(\cdot, t)-\chi_{i 2}(\cdot, t)\right\|_{L^{2}(\Omega)}^{2} \\
& \leq \bar{t}^{2} C_{8}\left\{\left\|\hat{\theta}_{1}-\hat{\theta}_{2}\right\|_{\left.L^{2}(\Omega \times] 0, \bar{t}\right)}^{2}+\left\|\hat{\chi}_{21}-\hat{\chi}_{22}\right\|_{\left.L^{2}(\Omega \times] 0, \bar{t}\right)}^{2}\right\},
\end{aligned}
$$


where, for instance, $C_{8}=6 C_{6}^{2}\left(C_{5}+1\right) \max \left\{1, C_{7}\right\}$. Thus, if $\bar{t}<1 / \sqrt{C_{8}}$, the operator $A$ defined by (3.27) is a contraction mapping in $X$ and we have one and only one solution of $(\mathrm{P})$ in $[0, \bar{t}]$. As we can start again and find the same estimate (with the same $C_{8}$ !) as in (3.37), the theorem is completely proved.

REMARK. If one neglects the fourth-order term $\nu \Delta$ (div u) in (3.4), then difficulties arise in reproducing the previous argument; more precisely it is no longer clear whether Lemma 1 holds.

As an alternative model, one can replace $\nu \Delta(\operatorname{div} \mathbf{u})$ with the term $\left\{-\operatorname{const} \Delta\left(\begin{array}{c}0 \\ \beta_{2}-\beta_{1}\end{array}\right)\right\}$ in the right-hand side of (3.5) (this can be justified from the physical point of view by assuming some diffusion effects). The new regularizing term allows to prove the existence of a solution by means of an approximation-a priori estimate-passage to the limit procedure. The uniqueness of the solution and its continuous dependence on the data can then be shown via a contraction technique, similar to that used for Theorem 1. However here we shall not detail this argument.

Acknowledgments. The authors are grateful to the referee for useful comments and suggestions.

\section{REFERENCES}

[1] M. Achenbach and I. Müller, Model for shape memory, J. Physique, C4 Suppl. 12, 43, 163-167 (1982)

[2] H. W. Alt, K. H. Hoffmann, M. Niezgodka, and J. Sprekels, A numerical study of structural phase transitions in shape memory alloys, Preprint No 90, Institut für Mathematik, Augsburg University, 1985

[3] S. Antman, J. L. Ericksen, D. Kinderlehrer, and I. Müller, eds., Metastability and Incompletely Posed Problems, Springer-Verlag, Berlin, 1987

[4] D. Blanchard, M. Frémond, and A. Visintin, Phase change with dissipation, Thermomechanical Coupling in Solids, H. D. Bui and Q. S. Nguyen eds., North Holland, 1987, pp. 411-418

[5] H. Brézis, Operatéurs maximaux monotones et semi-groupes de contractions dans les espaces de Hilbert, Math. Studies 5, North-Holland, Amsterdam-London, 1973

[6] H. Brézis, Problèmes unilatéraux, J. Math. Pures Appl. 51, 1-168 (1972)

[7] F. Falk, Martensitic domain boundaries in shape-memory alloys as solitary waves, J. Physique, C4 Suppl. 12, 43, 3-15 (1982)

[8] M. Frémond, Matériaux à mémoire de forme, C. R. Acad. Sci. Paris Sér. II Méc. Phys. Chim. Sci. Univers Sci. Terre 304, 239-244 (1987)

[9] M. Frémond and P. Nicolas, Hystérésis dans les milieux poreux humides non saturés, C. R. Acad. Sci. Paris Sér. II Méc. Phys. Chim. Sci. Univers Sci. Terre 305, 741-746 (1987)

[10] M. Frémond and A. Visintin, Dissipation dans le changement de phase. Surfusion. Changement de phase irréversible, C. R. Acad. Sci. Paris Sér. II Méc. Phys. Chim. Sci. Univers Sci. Terre 301, 1265$1268(1985)$

[11] P. Germain, La méthode des puissances virtuelles en mécanique des milieux continus $-1^{0}$ partie, Théorie du second gradient, J. Mécanique 12, 235-274 (1973)

[12] G. Guénin, Alliages à mémoire de forme, Technique de l'ingenieur, M 530, Paris, 1986

[13] G. Herget, M. Müllner, G. Eckold, H. Tietze, and W. Assmus, Strukturelle Phasenübergänge der Formgedächtnislegierung NiTi, Jahresbericht 1987 des Inst. für Kernphysik, p. 77, Johann Wolfgang Göthe Universität, Frankfurt/Main, 1987

[14] MSI Workshop: The mathematical analysis of material microstructure, Cornell University, June $15-18,1988$

[15] J. Nečas, Les méthodes directes en théorie des équations elliptiques, Academia, Prague, 1967

[16] M. Niezgodka and J. Sprekels, Existence of solutions for a mathematical model of structural phase transitions in shape memory alloys, Math. Methods Appl. Sci. 10, 197-223 (1988) 